\title{
Real-world use of PCSK-9 inhibitors by early adopters: cardiovascular risk factors, statin co-treatment, and short-term adherence in routine clinical practice
}

\author{
This article was published in the following Dove Press journal: \\ Therapeutics and Clinical Risk Management \\ 3 August 2017 \\ Number of times this article has been viewed
}

\section{Kathleen A Fairman \\ Lindsay E Davis \\ David A Sclar}

Department of Pharmacy Practice, College of Pharmacy, Midwestern University-Glendale, Glendale, AZ, USA
Correspondence: Kathleen A Fairman Department of Pharmacy Practice, College of Pharmacy, Midwestern University, 19555 N 59th Avenue, Glendale, AZ 85308, USA

Tel +l 4803569296

Email kfairm@midwestern.edu
Background: Inconsistency of real-world medication use with labeled indications may affect cost and clinical value of pharmacotherapy. PCSK-9 inhibitors are labeled in the US for use with statins to reduce low-density lipoprotein cholesterol in patients with atherosclerotic cardiovascular disease (ASCVD) or familial hypercholesterolemia (FH).

Objective: To assess consistency with labeled indications and treatment persistency for early (first 5 post-launch months) adopters of PCSK-9 inhibitor pharmacotherapy.

Methods: Retrospective analysis of commercially insured cohorts derived from the Truven Health MarketScan ${ }^{\circledR}$ database was performed. Subjects were aged 18-64 years, initiated PCSK-9 inhibitor or highest-intensity statin (rosuvastatin $40 \mathrm{mg}$ /day or atorvastatin $80 \mathrm{mg}$ /day) pharmacotherapy from August to December 2015, and were enrolled throughout 2015 and during separate baseline (pre-treatment) periods of 6 and 18 months. Baseline ASCVD, FH, and ASCVD events (myocardial infarction, transient ischemic attack, and cerebrovascular occlusion) were measured. Persistency was measured through December 2015 for subcohorts of patients initiating treatment from August to September 2015.

Results: Baseline disease rates were higher for patients treated with PCSK-9 inhibitors ( $\mathrm{n}=390)$ compared with highest-intensity statins ( $\mathrm{n}=26,306)$ : ASCVD (68.5\% vs 33.4\%, respectively); FH (39.7\% vs $15.5 \%$ ); both $P<0.001$. In 18 months pre-treatment, $35.6 \%$ of PCSK-9 inhibitortreated patients had $\geq 1$ ASCVD event, and $87.9 \%$ had a labeled indication. Rates of 60 -day nonpersistency for PCSK-9 inhibitors and highest-intensity statins were 33.3\% and 39.8\%, respectively $(P=0.207)$. During PCSK-9 inhibitor pharmacotherapy, $33.8 \%$ of patients had evidence of statin supply and, of those initiating treatment from August to September, $40.9 \%$ filled $\geq 1$ statin prescription. Of those with sustained pre-treatment statin use, $34.8 \%$ had no statin supply during PCSK-9 inhibitor pharmacotherapy.

Conclusion: Among early-adopting PCSK-9 inhibitor-treated patients, the off-label diagnosis rate was $12 \%$; a majority lacked statin co-treatment; and one third filled prescriptions for $\leq 60$ days. Inconsistency with labeled uses may reflect prescriber/patient decisions, healthinsurance coverage determinations, or statin intolerance not reported on claims.

Keywords: hyperlipidemia, PCSK-9 inhibitors, alirocumab, evolocumab, off-label use, specialty medications

\section{Introduction}

Two PCSK-9 inhibitors, alirocumab (sanofi-aventis U.S. LLC. Bridgewater, NJ, USA and Regeneron Pharmaceuticals, Inc. Tarrytown, NY, USA) and evolocumab (Amgen Inc. Thousand Oaks, CA), are currently approved by the US Food and Drug 
Administration (FDA) for use in two disease indications, in conjunction with dietary modification and "maximally tolerated statin therapy", to reduce low-density lipoprotein cholesterol (LDL-C) which remains above goal despite statin treatment. ${ }^{1,2}$ FDA-labeled disease indications for PCSK-9 inhibitors include clinical atherosclerotic cardiovascular disease (ASCVD) and familial hypercholesterolemia (FH), a genetic metabolic disorder affecting about $0.4 \%$ of adults. ${ }^{3,4}$ In Europe, the medications are approved for a somewhat broader list of diagnoses, including $\mathrm{FH}$, non-FH, and mixed dyslipidemia. $^{5}$

Although treatment with PCSK-9 inhibitors reduced LDL-C by an average of $65.29 \mathrm{mg} / \mathrm{dL}$ in randomized controlled trials, ${ }^{6}$ market launch of the drugs in the US was accompanied by considerable questioning about their use in routine clinical practice, based on both economic and clinical factors. ${ }^{7,8}$ The foremost economic consideration was the high acquisition cost for PCSK-9 inhibitors, estimated at US\$12,000-US\$14,000 annually, compared with less than US\$100 annually for generic statins..$^{8-10}$

A related consideration was the high prevalence of hypercholesterolemia, which affects approximately $28 \%$ of US adults. ${ }^{11}$ Specifically, industry observers and employer coalitions suggested that off-label use, particularly in patients with hypercholesterolemia but without ASCVD or FH, could dramatically expand treatment prevalence, increase total drug costs to unsustainable levels, and eventually result in curtailing or termination of employer-based health care benefits. ${ }^{12,13}$

Clinical considerations included the need for parenteral administration, lack of sufficiently powered long-term studies of safety and cardiovascular outcomes, and questions about whether real-world use of PCSK-9 inhibitors would be consistent with clinical trial protocols. ${ }^{7,12,14,15}$ Specifically, the FDA Endocrinologic and Metabolic Drugs Advisory Committee expressed concern that patients who were "not truly intolerant" of statins would use PCSK-9 inhibitors as monotherapy despite the use of background statin therapy in most patients studied in clinical trials..$^{1,2,14,15}$ Conversely, some observers suggested that compared with daily oral administration of statins, once- or twice-monthly injections of PCSK-9 inhibitors had the potential to improve therapy adherence, thereby reducing the rate of cardiovascular events. ${ }^{16}$

To date, no studies of which these authors are aware have examined how and by whom PCSK-9 inhibitors are being used in routine clinical practice. To address this need, the present study, conducted using a large database of commercially insured enrollees, examined real-world use of PCSK-9 inhibitors in the first 5 post-launch months. Examination of patients treated immediately after launch is important in a therapy class with the potential for off-label use or an adherence advantage, because early adopters of innovative therapies may represent those with the most immediate clinical needs. ${ }^{17}$ Study metrics included 1) baseline (pretreatment) rates of cardiovascular risk factors, indications for use, and comorbidities; 2) concomitant use of other lipid-lowering medications; and 3) treatment persistency.

\section{Methods}

\section{Data source and design}

The study was a retrospective analysis of cohorts derived from the Truven Health MarketScan ${ }^{\circledR}$ Commercial Claims and Encounters database. The database is a fully deidentified, Health Insurance Portability and Accountability Act (HIPAA)-compliant dataset comprising claims for all health care (medical and pharmacy) services provided to approximately 50 million commercially insured enrollees each year. It has been used in more than 1,400 published studies of US health care, including numerous analyses of commercially insured enrollees with cardiovascular diseases and related comorbidities. ${ }^{18-22}$ Data are obtained by Truven Health from employers and health insurance plans, cleaned for quality and accuracy, and de-identified using encrypted case numbers for research purposes. The study was deemed exempt from Institutional Review Board (IRB) review by the Midwestern University IRB committee.

The study compared patients newly treated with PCSK-9 inhibitors vs highest-intensity statin therapy, defined as a rosuvastatin (AstraZeneca Pharmaceuticals. Wilmington, DE, USA) dosage of $40 \mathrm{mg} /$ day or atorvastatin dosage of $80 \mathrm{mg} /$ day. ${ }^{23}$ Highest-intensity statin treatment was used as a comparator for two reasons. First, rates of nonadherence to statin pharmacotherapy are high in routine clinical practice. ${ }^{24,25}$ Second, high-intensity statins are a potential treatment alternative when lower-dose statin therapy does not achieve the desired level of LDL-C reduction. ${ }^{26}$

The term "highest-intensity" is used throughout this article because treatment guidelines identify "high-intensity" statin therapy in daily dosage ranges of $40-80 \mathrm{mg}$ atorvastatin or 20-40 mg rosuvastatin. ${ }^{26}$ The top of the range was chosen for cohort identification in the present study because one would expect greater statin discontinuation rates at higher dosages, ${ }^{27}$ thereby providing a conservative point of comparison for rates of PCSK-9 inhibitor discontinuation. To test the effect of this decision, a sensitivity analysis examined patients treated with atorvastatin $\geq 40 \mathrm{mg} /$ day or rosuvastatin $\geq 20 \mathrm{mg} /$ day. Statin dosages per day were 
calculated as total mg dispensed (strength per unit multiplied by number of units), divided by the days supply field value reported on the claim.

\section{Sampling and cohort identification process}

All subjects meeting the criteria listed below were included in the study sample. Included subjects: 1) were aged 18-64 years and continuously enrolled for benefits throughout 2015 $(\mathrm{n}=15,448,306) ; 2)$ newly initiated pharmacotherapy with a study medication regimen - either a) a PCSK-9 inhibitor, with or without one or more statins (PCSK-9 inhibitor cohort, $\mathrm{n}=390$ ) or b) highest-intensity statin therapy without a PCSK-9 inhibitor (statin cohort, $n=26,306$ ) - during the time period from August 1, 2015, to December 31, 2015. New initiation was defined as filling $\geq 1$ prescription for a regimen beginning at any time on or after August 1, 2015, with no (zero) filled prescriptions for that regimen from January 1, 2015, to July 31, 2015. This design was chosen because of the August 2015 market launch of PCSK-9 inhibitors. In both cohorts, a proportion of patients used one or more statins prior to initiating the study medication regimen, as described in the following section "Measurement of pre-treatment characteristics".

\section{Measurement of pre-treatment characteristics}

For each sampled subject, analyses of baseline demographic and clinical characteristics were performed. Clinical characteristics were measured during two separate baseline periods comprising the 183 days (6-month pre-treatment baseline period) and 548 days (18-month pre-treatment baseline period) prior to the first observed study medication claim (PCSK-9 inhibitor or highest-intensity statin). The 18-month analysis was limited to subcohorts of subjects who were continuously enrolled throughout both 2014 and 2015 (n=21,563 highest-intensity statin subcohort; n=340 PCSK-9 inhibitor subcohort). Baseline clinical characteristics included diagnoses of ASCVD and FH; ASCVD events indicating use in secondary prevention (ie, myocardial infarction, transient ischemic attack [TIA], and cerebrovascular occlusion); potential statin intolerance; and comorbidities.

Diagnoses were measured in any of the first four diagnosis fields reported on ambulatory claims (outpatient hospital department, emergency department, and physician office), and in these four fields plus the primary diagnosis field and diagnosis-related group (DRG) codes on inpatient hospital claims. Revascularization, including percutaneous transluminal angioplasty, stenting, balloon angioplasty, and coronary artery bypass grafting, was measured using procedure codes for all treatment settings and DRG codes for inpatient admissions.

Because no specific International Classification of Diseases (ICD) codes are available for FH or statin intolerance, proxy measures were used. In accordance with guidance provided on instructional websites for claims (billing) coders and descriptions provided in the ICD-9 and ICD-10, FH was defined as "pure hypercholesterolemia" and coded as ICD-9=272.0x or ICD-10=E78.0x. ${ }^{28-30}$ For statin intolerance, which is defined as clinically significant myalgia, cognitive impairment, or rhabdomyolysis, a number of coding approaches ranging in scope have been tested. ${ }^{31,32}$ In the present study, statin intolerance was defined as diagnoses of rhabdomyolysis (ICD-728.88 or ICD-10=M62.82), unspecified adverse effect of drug (ICD-9=995.2), or adverse effects of cardiovascular drugs or unspecified drugs (ICD-10=T46 or T50). As a validation metric for this approach, the resulting prevalence rate was compared to the known prevalence rate for statin intolerance, which is estimated at $1 \%-10 \%{ }^{31}$

Pre-treatment statin utilization was assessed for the time period beginning on January 1, 2015, and ending on the day prior to the start of the study medication regimen. Three metrics were assessed: 1 ) any statin use, defined as $\geq 1$ claim for any statin at any dosage; 2 ) $\geq 1$ claim for $\geq 20 \mathrm{mg}$ rosuvastatin or $\geq 40 \mathrm{mg}$ atorvastatin daily (ie, the lower end of the range considered "high-intensity" in treatment guidelines); ${ }^{26}$ and 3 ) sustained statin use, defined as a total of $\geq 120$ days supply or $\geq 3$ claims.

\section{Measurement of pharmacotherapy characteristics}

The assessment of pharmacotherapy characteristics comprised two metrics: adherence and use of other lipid-lowering pharmacotherapy in addition to study medications. Because a history of cardiovascular events may affect both of these metrics, they were measured both for the sample overall and for the secondary-prevention subcohort.

Adherence to pharmacotherapy with either highestintensity statins or a PCSK-9 inhibitor was measured as shortterm persistence, defined as the number of days from the first to the final study medication claim fill dates in calendar year 2015. To permit a sufficient measurement time frame for the reporting of 60-day persistency rates, this analysis was limited to patients whose first study medication claim (ie, either for highest-intensity statin therapy or for PCSK-9 inhibitor therapy) was in August or September of 2015, yielding a minimum of 90 days follow-up. 
Concomitant statin use during PCSK-9 inhibitor treatment was assessed using two metrics. First, for all PCSK-9 inhibitor-treated patients, concomitant use was defined as either filling $\geq 1$ prescription for a statin after the start of the study medication or having available medication supply for $\geq 30$ days after the start of treatment. The 30 -day requirement was chosen as a minimal measure of statin use, because the availability of 30 days of drug supply is a common metric in research using pharmacy claims data, and because in previous studies of concomitant pharmacotherapies, results for 30-, 60-, and 90-day overlap were generally similar. ${ }^{33-35}$ For this metric, medication supply after treatment start was calculated by 1) summing the fill date plus the days supply for the final pre-treatment statin claim to produce a pretreatment medication depletion date, then 2) calculating the number of days from the treatment start date to the pretreatment medication depletion date. For example, a patient who filled a prescription for a 90-day supply of a statin on November 1, 2015, then initiated PCSK-9 inhibitor treatment on December 15, 2015, would be assumed to have available statin supply until January 30, 2016 (ie, 46 days after treatment start), thereby meeting the 30-day requirement.

Second, for patients whose first study medication claim was in August or September 2015, concomitant use was defined as filling $\geq 1$ statin prescription at any time after the start of PCSK-9 inhibitor treatment. The rationale for this metric was that in a minimum of 90 days of follow-up for a patient using a PCSK-9 inhibitor and statin concomitantly, one would expect $\geq 1$ filled statin prescription.

Bivariate analyses of patient characteristics used an alpha (critical $P$ ) value of 0.05 , with Student's $t$-tests for interval-scale variables and Pearson chi-square tests for categorical variables. To produce nationally representative estimates, all results were weighted for the sample-topopulation ratio across strata formed on sex, age group, region, and policy-holder status (ie, enrollee vs dependent), using a method and strata population sizes provided by Truven Health. The total sample size after weighting was held to the original (pre-weighting) cohort size by applying a constant to all strata weights. All statistical analyses were performed with SPSS (IBM Corporation, Armonk, NY, USA) version 23.0.

\section{Results}

\section{Pre-treatment characteristics}

Cohorts of patients newly treated with PCSK-9 inhibitor $(n=390)$ and highest-intensity statin $(n=26,306)$ pharmacotherapy did not significantly differ with respect to sex or baseline rates of chronic kidney disease, cerebral occlusion/
TIA, or myocardial infarction measured in the 6-month pre-treatment period (Table 1). Secondary-prevention rates were similar for patients treated with PCSK-9 inhibitors and highest-intensity statins (21.0 vs $20.0 \%$, respectively; $P=0.612$ ). However, rates of ASCVD prevalence were higher for patients treated with PCSK-9 inhibitors $(68.5 \%)$ compared with highest-intensity statins $(33.4 \%, P<0.001)$. PCSK-9 inhibitor-treated patients were approximately 2 years older, on average, than those treated with highestintensity statin pharmacotherapy (means of 55 vs 53 years, respectively; $P<0.001)$.

When measured in the 18 months prior to initiation of study medication, prevalence rates for cardiovascular disease, most comorbidities, and cardiovascular events were generally higher for patients treated with PCSK-9 inhibitors $(n=340)$ than for those treated with highest-intensity statins $(n=21,563$; Table 1). Nearly one half of PCSK-9 inhibitor-treated patients (48.8\%), compared with $25.5 \%$ of those treated with highestintensity statins, had a documented/coded diagnosis of FH $(P<0.001)$. Those treated with PCSK-9 inhibitors were also more likely than were highest-intensity statin-treated patients to have had a prior ASCVD event (ie, secondary prevention, $35.6 \%$ vs $24.8 \%$, respectively; $P<0.001$ ).

Characteristics of the patient population treated with PCSK-9 inhibitors deviated somewhat from labeled indications (Table 1). The 6-month baseline prevalence rate on a composite metric of ASCVD or FH (ie, labeled indications) was $81.0 \%$ for PCSK-9 inhibitor-treated patients. In the 18-month baseline period, prevalence rates for the composite of ASCVD or FH were $87.9 \%$ and $54.5 \%$ for patients treated with PCSK-9 inhibitors and highest-intensity statins, respectively $(P<0.001)$.

Patients receiving PCSK-9 inhibitor pharmacotherapy were somewhat more likely than were highest-intensity statin-treated patients to have used any statin $(59.5 \%$ vs $52.4 \%$, respectively; $P=0.006$ ) or rosuvastatin of $\geq 20 \mathrm{mg}$ or atorvastatin of $\geq 40 \mathrm{mg}$ daily (33.3\% vs $26.3 \%$, respectively; $P=0.002$ ) prior to initiating the study medication regimen. Rates of highest-intensity statin use in the pre-treatment period were $24.4 \%$ in the PCSK-9 inhibitor cohort and, by design, $0 \%$ in the highest-intensity statin cohort. Rates of sustained pre-treatment statin use ( $\geq 120$ days supply or $\geq 3$ claims) for the two cohorts did not significantly differ $(47.7 \%$ vs $42.8 \%$, respectively; $P=0.052$ ).

\section{Pharmacotherapy characteristics}

Approximately one third $(33.8 \%)$ of patients treated with PCSK-9 inhibitors had evidence of statin medication supply during treatment, based on prescriptions filled in either the 
Table I Baseline demographic and clinical characteristics, patients initiating pharmacotherapy with highest-intensity statin or PCSK-9 inhibitor, August through December 2015

\begin{tabular}{|c|c|c|c|}
\hline Patient characteristics & $\begin{array}{l}\text { Highest-intensity } \\
\text { statin }^{\text {a }}\end{array}$ & $\begin{array}{l}\text { PCSK-9 } \\
\text { inhibitor }\end{array}$ & $P$-value \\
\hline$\overline{\mathrm{N}}$ & 26,306 & 390 & - \\
\hline Age mean (median) & $53(55)$ & $55(57)$ & $<0.001$ \\
\hline Female (\%) & 34.6 & 38.7 & 0.089 \\
\hline \multicolumn{4}{|l|}{ Pre-treatment statin use in calendar year $2015(\%)^{\mathrm{b}}$} \\
\hline Any use ( $\geq$ I claim) & 52.4 & 59.5 & 0.006 \\
\hline Sustained use ( $\geq 120$ days supply and/or $\geq 3$ claims) & 42.8 & 47.7 & 0.052 \\
\hline Rosuvastatin $\geq 20 \mathrm{mg}$ or atorvastatin $\geq 40 \mathrm{mg}$ daily ( $\geq$ I claim) & 26.3 & 33.3 & 0.002 \\
\hline Highest-intensity statin ${ }^{a}(\geq$ I claim) & 0.0 & 24.4 & - \\
\hline \multicolumn{4}{|l|}{ Diagnoses in 6-month pre-treatment baseline } \\
\hline Chronic kidney disease (\%) & 2.4 & 2.8 & 0.604 \\
\hline Diabetes (\%) & 30.3 & 22.1 & $<0.001$ \\
\hline Hypertension (\%) & 53.0 & 64.6 & $<0.001$ \\
\hline Hypercholesterolemia (\%) & 62.9 & 92.8 & $<0.001$ \\
\hline Familial hypercholesterolemia (\%) & 15.5 & 39.7 & $<0.001$ \\
\hline Statin intolerance (\%) & 0.4 & 3.6 & $<0.001$ \\
\hline \multicolumn{4}{|l|}{ ASCVD (\%) } \\
\hline Angina & 5.7 & 11.3 & $<0.001$ \\
\hline $\mathrm{CABG} / \mathrm{PTCl}$ & 10.7 & 5.4 & 0.001 \\
\hline Cerebral occlusion/TIA & 8.3 & 9.7 & 0.312 \\
\hline Myocardial infarction & 12.9 & 13.3 & 0.780 \\
\hline $\mathrm{PAD} /$ extremity revascularization & 2.5 & 6.9 & $<0.001$ \\
\hline Any ASCVD ${ }^{c}$ & 33.4 & 68.5 & $<0.001$ \\
\hline ASCVD or familial hypercholesterolemia (PCSK-9 inhibitor indications) & 42.4 & 81.0 & $<0.001$ \\
\hline ASCVD event (secondary prevention) ${ }^{d}$ & 20.0 & 21.0 & 0.612 \\
\hline \multicolumn{4}{|l|}{ Diagnoses in 18 -month pre-treatment baseline $e^{e}$} \\
\hline $\mathrm{N}$ & 21,563 & 340 & \\
\hline Chronic kidney disease (\%) & 3.5 & 3.5 & 0.941 \\
\hline Diabetes (\%) & 35.3 & 25.0 & $<0.001$ \\
\hline Hypertension (\%) & 67.0 & 77.1 & $<0.001$ \\
\hline Hypercholesterolemia (\%) & 80.7 & 96.5 & $<0.001$ \\
\hline Familial hypercholesterolemia (\%) & 25.5 & 48.8 & $<0.001$ \\
\hline Statin intolerance $(\%)$ & 0.9 & 6.5 & $<0.001$ \\
\hline \multicolumn{4}{|l|}{ ASCVD (\%) } \\
\hline Angina & 7.7 & 19.7 & $<0.001$ \\
\hline $\mathrm{CABG} / \mathrm{PTCl}$ & 12.5 & 10.9 & 0.384 \\
\hline Cerebral occlusion/TIA & 11.4 & 21.4 & $<0.001$ \\
\hline Myocardial infarction & 15.5 & 19.7 & 0.032 \\
\hline $\mathrm{PAD} /$ extremity revascularization & 4.0 & 9.7 & $<0.001$ \\
\hline Any ASCVD ${ }^{c}$ & 40.8 & 77.4 & $<0.001$ \\
\hline ASCVD or familial hypercholesterolemia (PCSK-9 inhibitor indications) & 54.5 & 87.9 & $<0.001$ \\
\hline ASCVD event (secondary prevention) ${ }^{d}$ & 24.8 & 35.6 & $<0.001$ \\
\hline
\end{tabular}

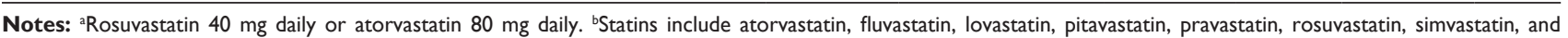
combination therapies including atorvastatin + amlodipine, lovastatin + niacin, simvastatin + ezetimibe, and simvastatin + niacin. Measured from January I, 20I5, through I day prior to the study medication start date. 'Any of the ASCVD diagnoses listed above or any code for atherosclerosis or ischemic heart disease. ${ }^{\mathrm{d} C e r e b r a l ~ o c c l u s i o n, ~ M I, ~ o r ~ T I A . ~}$ eMeasurement of I8-month baseline diagnoses was limited to patients continuously enrolled through 2014 and 2015.

Abbreviations: ASCVD, atherosclerotic cardiovascular disease; CABG, coronary artery bypass grafting; MI, myocardial infarction; PAD, peripheral arterial disease; PTCI, percutaneous transluminal coronary intervention; TIA, transient ischemic attack.

pre-treatment period or after the start of PCSK-9 pharmacotherapy (Table 2). Among patients initiating PCSK-9 inhibitor pharmacotherapy in August or September, 40.9\% filled $\geq 1$ statin prescription prior to December 31, 2015. However, PCSK-9 inhibitor-treated patients were much more likely to fill $\geq 1$ prescription for ezetimibe than were those treated with highest-intensity statins ( $16.7 \%$ vs $2.4 \%$, respectively; $P<0.001)$. Of patients identified as sustained statin users in the pre-treatment period who subsequently used a PCSK-9 inhibitor ( $\mathrm{n}=187), 65.2 \%$ had evidence of statin medication supply during PCSK-9 inhibitor pharmacotherapy (not shown in Table 2). 
Table 2 Characteristics of pharmacotherapy with highest-intensity statins or PCSK-9 inhibitors, patients initiating treatment from August through December, 2015

\begin{tabular}{|c|c|c|c|}
\hline Whole sample & $\begin{array}{l}\text { Highest-intensity } \\
\text { statin }^{\mathrm{a}}\end{array}$ & $\begin{array}{l}\text { PCSK-9 } \\
\text { inhibitor }\end{array}$ & $P$-value \\
\hline \multicolumn{4}{|l|}{ Use of other lipid-lowering therapies, \% of patients } \\
\hline $\mathrm{N}$ & 26,306 & 390 & - \\
\hline Any statin use $\mathrm{e}^{\mathrm{b}}$ & Inapplicable ${ }^{c}$ & 33.8 & - \\
\hline Statin, $\geq$ I prescription filled, patients initiating & Inapplicable ${ }^{c}$ & 40.9 & - \\
\hline \multicolumn{4}{|l|}{ PCSK-9 inhibitor treatment in August or September ${ }^{d}$} \\
\hline Ezetimibe, $\geq$ I prescription filled ${ }^{e}$ & 2.4 & 16.7 & $<0.001$ \\
\hline Prescription-strength fish oil, $\geq$ I prescription filled & 0.1 & 0.3 & 0.148 \\
\hline \multicolumn{4}{|c|}{ Duration, patients initiating treatment in August or September,f } \\
\hline Mean (SD) days & $63(47)$ & $69(4 I)$ & 0.183 \\
\hline 60 days or less, $\%$ & 39.8 & 33.3 & 0.207 \\
\hline \multicolumn{3}{|c|}{ Number of claims, subjects initiating treatment in August or September, \% } & $<0.001$ \\
\hline I & 29.3 & 15.1 & \\
\hline 2 & 31.6 & 18.3 & \\
\hline 3 & 15.3 & 9.7 & \\
\hline 4 or more & 23.8 & 57.0 & \\
\hline \multicolumn{4}{|c|}{ Secondary-prevention subcohort: subjects with cerebral occlusion, TIA, or MI in I8-month pre-treatment baseline } \\
\hline \multicolumn{4}{|l|}{ Use of other lipid-lowering therapies, \% of patients } \\
\hline $\mathrm{N}$ & 5,302 & 117 & - \\
\hline Any statin use ${ }^{b}$ & Inapplicable ${ }^{c}$ & 40.2 & - \\
\hline Statin, $\geq$ I prescription filled, patients initiating & Inapplicable ${ }^{c}$ & 51.9 & - \\
\hline \multicolumn{4}{|l|}{ PCSK-9 inhibitor treatment in August or September ${ }^{d}$} \\
\hline Ezetimibe, $\geq$ I prescription filled ${ }^{e}$ & 2.5 & 14.0 & $<0.001$ \\
\hline Prescription-strength fish oil, $\geq$ I prescription filled & 0.1 & 0.0 & 0.794 \\
\hline \multicolumn{4}{|c|}{ Duration, patients initiating treatment in August or September ${ }^{\mathrm{d}, \mathrm{f}}$} \\
\hline Mean (SD) days & $71(44)$ & $69(36)$ & 0.817 \\
\hline 60 days or less, $\%$ & 34.2 & 44.4 & 0.266 \\
\hline \multicolumn{4}{|c|}{ Number of claims, patients initiating treatment in August or September, \% } \\
\hline 1 & 19.7 & II.I & 0.161 \\
\hline 2 & 25.2 & 33.3 & \\
\hline 3 & 20.1 & 7.4 & \\
\hline 4 or more & 35.0 & 48.1 & \\
\hline
\end{tabular}

Notes: 'Rosuvastatin $40 \mathrm{mg}$ daily or atorvastatin $80 \mathrm{mg}$ daily. ${ }^{b}$ Indicates that during pharmacotherapy with the PCSK-9 inhibitor, patient either had $\geq 30$ days of statin supply, based on the depletion date (fill date plus days supply) for the final pre-treatment statin claim, or filled $\geq 1$ statin prescription. 'By design, $100 \%$ of patients in this group met the criterion shown in the row label. 'Subcohort of the whole sample: $n=8,686$ highest-intensity statin; $n=93$ PCSK-9 inhibitor. Subcohort of the secondary-prevention subcohort: $n=1,955$ highest-intensity statin; $n=27$ PCSK-9 inhibitor. ${ }^{e}$ Either alone or in combination with simvastatin. 'Number of days from first to final fill date. Abbreviations: MI, myocardial infarction; TIA, transient ischemic attack.

In the secondary-prevention subcohort of PCSK-9 inhibitor-treated patients (ie, those with $\geq 1$ ASCVD event in the 18-month pre-treatment baseline period), the rate of statin co-treatment was somewhat higher (ie, $40.2 \%$ with evidence of medication supply and $51.9 \%$ filling $\geq 1$ statin prescription). Within this subcohort, $14.0 \%$ used ezetimibe concomitantly with the PCSK-9 inhibitor.

Among those initiating treatment in August or September 2015, mean durations of therapy prior to December 31, 2015, were similar for patients treated with PCSK-9 inhibitors (69 days) and highest-intensity statins (63 days; $P=0.183$; Table 2). In the sensitivity analysis of patients treated with atorvastatin $\geq 40 \mathrm{mg} /$ day or rosuvastatin $\geq 20 \mathrm{mg} /$ day $(n=65,645)$, mean duration of statin therapy was 126 days (not shown in Table 2).
Approximately one third of PCSK9-treated patients filled only one or two claims, and $33.3 \%$ became nonpersistent with treatment after 60 days. In the secondaryprevention subcohort, mean duration of PCSK-9 inhibitor treatment was 69 days, and $44.4 \%$ were treated for 60 days or less.

\section{Discussion}

This retrospective cohort analysis of patients newly treated with highest-intensity statin or PCSK-9 inhibitor pharmacotherapy identified suboptimal treatment patterns, including potentially off-label use rates of $19 \%$ and $12 \%$ based on the 6- and 18-month periods, respectively, prior to treatment initiation; a monotherapy rate of 59\%-66\%; and a 60 -day nonpersistency rate of $33 \%$. The rate of statin co-treatment 
was somewhat higher, but still suboptimal, when PCSK-9 inhibitors were used in secondary prevention.

In light of complaints from physicians and the pharmaceutical industry about inappropriate coverage denials for patients in need of PCSK-9 inhibitor treatment, ${ }^{36-38}$ it is important to note that the present study was limited to patients treated with PCSK-9 inhibitors and did not assess the important question of whether patients meeting labeled indications received PCSK-9 inhibitor pharmacotherapy. The present study findings, coupled with a recent preliminary report that PCSK-9 inhibitor coverage denials are more common among patients with LDL-C exceeding $190 \mathrm{mg} / \mathrm{dL}$ than among those with lower LDL-C levels, ${ }^{39}$ suggest that PCSK-9 inhibitor treatment and/or coverage in the immediately post-launch phase may have been suboptimally targeted.

In interpreting these findings, it is important to note that whether treatment decisions were made primarily by prescribers and patients, or determined by health-insurance coverage decisions, cannot be ascertained from the present study data. It is possible that the unexpectedly high rate of 60-day discontinuation observed in this study is the result of prior authorization requirements for periodic assessments of treatment response, although coverage policies generally call for reassessments after 3 to 12 months of treatment - not after only 30 to 60 days. $^{40-43}$

\section{Limitations}

Several limitations and directions for future research should be noted. First, it was not possible to measure cardiovascular outcomes, as the probabilities of serious cardiovascular events (eg, myocardial infarction, revascularization) are so low that the number of events expected in a 5-month time frame would be extremely small. ${ }^{44,45}$ Future research should examine real-world outcomes for these patient populations, with a focus on number needed to treat and cost to prevent a major cardiac event with PCSK-9 inhibitor vs highest-intensity statin therapy. Assessment of total drug cost for these analyses should include patients who initiated treatment with either PCSK-9 inhibitors or statins, but were nonpersistent and therefore did not receive long-term clinical benefit.

Second, a portion of the unexpectedly high rate of PCSK-9 inhibitor monotherapy may have been attributable to statin intolerance which was not recorded in the claims, either because of a lack of specific diagnosis code or because of symptoms that occurred prior to the study time frame (eg, during a time period of coverage by a different insurer or employer). It is also possible that the statin monotherapy rate reflects confounding by indication; specifically, the targeting of PCSK-9 inhibitor treatment to patients who are statin intolerant but need LDL-C reduction.

However, it seems unlikely that the high rate of monotherapy observed in the present study is due entirely to statin intolerance, as the prevalence of true statin intolerance is estimated at $1 \%-10 \%$, consistent with the rate identified in the present study. ${ }^{31}$ Moreover, among the PCSK-9 inhibitor cohort, approximately one third of sustained statin users in the pre-treatment period had no evidence of statin supply during PCSK-9 inhibitor treatment. This finding suggests that these patients may have switched from a statin to a PCSK-9 inhibitor despite the ability to tolerate statins for a sustained period of time.

Third, it is possible that patients filled prescriptions for generic statins using a discount program, without presenting their insurance card. ${ }^{46,47}$ If so, statin use in the present study was underestimated.

Finally, the sample was limited to early adopters, whose characteristics may differ from those of patients treated later in the product life-cycle. In particular, one pharmaceutical manufacturer launched a televised advertising campaign for evolocumab in March 2017, and our findings may not represent patients newly treated after that date. ${ }^{48}$ Additionally, to provide information relevant to plans providing employer-based coverage, which have been the subject of the greatest concern about PCSK-9 inhibitor utilization and cost in the US, ${ }^{13}$ the present study sample was limited to commercially insured enrollees with a maximum age of 64 years. Similar research should be conducted in Medicare and Medicaid.

\section{Conclusion}

A retrospective cohort analysis of early-adopting PCSK-9 inhibitor-treated commercially insured enrollees found an off-label use rate of $12 \%$ when diagnoses up to 18 months prior to treatment were measured; a 59\%-66\% rate of monotherapy without statin co-treatment, despite a documented statin intolerance rate of $<10 \%$; and a $33 \%$ rate of 60 -day discontinuation. Future research should measure real-world use patterns in Medicare and Medicaid, as well as pharmacoeconomic outcomes, including the occurrence and cost of cardiovascular events.

\section{Disclosure}

Kathleen A Fairman discloses that she is a health outcomes research consultant whose analyses include assessments 
of specialty medications; however, she has no relationships with manufacturers of any lipid-lowering products and received no funds or other support for the present study, other than that provided by Midwestern University. Lindsay E Davis and David A Sclar have no financial conflicts of interest to disclose. This study was funded entirely by Midwestern University with no external sponsorship. The authors report no other conflicts of interest in this work.

\section{References}

1. Repatha ${ }^{\circledR}$ (evolocumab) injection, for subcutaneous use [package insert]. Thousand Oaks, CA: Amgen; 2015.

2. Praluent ${ }^{\mathbb{}}$ (alirocumab) injection, for subcutaneous use [package insert]. Bridgewater, NJ: Sanofi-Aventis; 2017.

3. Goldberg AC, Gidding SS. Knowing the prevalence of familial hypercholesterolemia matters. Circulation. 2016;133(11):1054-1057.

4. Reiner Z. Management of patients with familial hypercholesterolemia. Nat Rev Cardiol. 2015;12(10):565-575.

5. van den Heuvel TW, Cohen AF, Rissman R. European drug market entries 2015 with new mechanisms of action. Clin Med (Lond). 2016; 16(5):475-480.

6. Li C, Lin L, Zhang W, et al. Efficacy and safety of proprotein convertase subtilisin/kexin 9 monoclonal antibody on hypercholesterolemia: a meta-analysis of 20 randomized controlled trials. J Am Heart Assoc. 2015;4(6):e001937.

7. Cainzos-Achirica M, Martin SS, Cornell JE, Mulrow CD, Guallar E. PCSK9 inhibitors: a new era in lipid-lowering treatment? Ann Intern Med. 2015;163(1):64-65.

8. The Wall Street Journal [homepage on the Internet]. Silverman E. New cholesterol meds may lower heart risks, but will they break the bank? Wall Street Journal; 2015 March 20. Available from: https:// blogs.wsj.com/pharmalot/2015/03/20/new-cholesterol-meds-maylower-heart-risks-but-will-they-break-the-bank/. Accessed July 10, 2017.

9. Arrieta A, Page TF, Veledar E, Nasir K. Economic evaluation of PCSK9 inhibitors in reducing cardiovascular risk from health system and private payer perspectives. PLoS One. 2017;12(1):e0169761.

10. Kazi DS, Moran AE, Coxson PG, et al. Cost-effectiveness of PCSK9 inhibitor therapy in patients with heterozygous familial hypercholesterolemia or atherosclerotic cardiovascular disease. JAMA. 2016; 316(7):743-753.

11. National Center for Health Statistics. Health, United States, 2015: With Special Feature on Racial and Ethnic Health Disparities. Hyattsville, MD; 2016.

12. Health Affairs Blog [homepage on the Internet]. Shrank W, Lotvin A, Singh S, Brennan T. In the debate about cost and efficacy, PCSK9 inhibitors may be the biggest challenge yet. Health Affairs Blog; 2015 February 17. Available from: http://healthaffairs.org/blog/2015/02/17/ in-the-debate-about-cost-and-efficacy-pcsk9-inhibitors-may-be-thebiggest-challenge-yet/. Accessed July 10, 2017.

13. Mehr SR. Will the PCSK9 inhibitors be employers' "line in the sand"? Am Health Drug Benefits. 2016;9(3):171-174.

14. U.S. Food and Drug Administration, Center for Drug Evaluation and Research. The Endocrinologic and Metabolic Drugs Advisory Committee Meeting. Briefing document: BLA 125559. Praluent (alirocumab) injection; FDA; 2015. Available from: https://www.fda.gov/ downloads/advisorycommittees/committeesmeetingmaterials/drugs/ endocrinologicandmetabolicdrugsadvisorycommittee/ucm449865.pdf. Accessed July 10, 2017.
15. U.S. Food and Drug Administration, Center for Drug Evaluation and Research. Summary minutes of the Endocrinologic and Metabolic Drugs Advisory Committee Meeting. FDA; 2015. Available from: https:// www.fda.gov/downloads/AdvisoryCommittees/CommitteesMeeting Materials/Drugs/EndocrinologicandMetabolicDrugsAdvisoryCommittee/UCM457467.pdf. Accessed July 10, 2017.

16. Institute for Clinical and Economic Review. PCSK9 inhibitors for treatment of high cholesterol: effectiveness, value, and value-based price benchmarks. Boston, MA: ICER; 2015. https://icer-review.org/ wp-content/uploads/2016/01/Final-Report-for-Posting-11-24-15-1.pdf. Accessed July 19, 2017.

17. Lubloy A. Factors affecting the uptake of new medicines: a systematic literature review. BMC Health Serv Res. 2014;14:469.

18. Truven Health Analytics. MarketScan studies: abbreviated bibliography. Truven Health Analytics; 2017. Available from: http://sites. truvenhealth.com/bibliography/TruvenHealthMarketScanBibliography. pdf. Accessed July 10, 2017.

19. Bonafede MM, Johnson BH, Richhariya A, Gandra SR. Medical costs associated with cardiovascular events among high-risk patients with hyperlipidemia. Clinicoecon Outcomes Res. 2015;7:337-345.

20. Lip GY, Hunter TD, Quiroz ME, Ziegler PD, Turakhia MP. Atrial fibrillation diagnosis timing, ambulatory ECG monitoring utilization, and risk of recurrent stroke. Circ Cardiovasc Qual Outcomes. 2017;10(1): pii: e002864.

21. Hwang AY, Dave C, Smith SM. Trends in antihypertensive medication use among US patients with resistant hypertension, 2008 to 2014. Hypertension. 2016;68(6):1349-1354.

22. Fitch K, Weisman T, Engel T, et al. Longitudinal commercial claimsbased cost analysis of diabetic retinopathy screening patterns. Am Health Drug Benefits. 2015;8(6):300-308.

23. Writing Committee, Lloyd-Jones DM, Morris PB, et al. 2016 ACC expert consensus decision pathway on the role of non-statin therapies for LDL-cholesterol lowering in the management of atherosclerotic cardiovascular disease risk: a report of the American College of Cardiology Task Force on Clinical Expert Consensus Documents. J Am Coll Cardiol. 2016;68(1):92-125.

24. Ellis JJ, Erickson SR, Stevenson JG, Bernstein SJ, Stiles RA, Fendrick MA. Suboptimal statin adherence and discontinuation in primary and secondary prevention populations: should we target patients with the most to gain? J Gen Intern Med. 2004;19(6):638-645.

25. Perreault S, Blais L, Lamarre D, et al. Persistence and determinants of statin therapy among middle-aged patients for primary and secondary prevention. Br J Clin Pharmacol. 2005;59(5):564-573.

26. Stone NJ, Robinson JG, Lichtenstein AH, et al. 2013 ACC/AHA guideline on the treatment of blood cholesterol to reduce atherosclerotic cardiovascular risk in adults: a report of the American College of Cardiology/American Heart Association Task Force on Practice Guidelines. J Am Coll Cardiol. 2014;63(25 Pt B):2889-2934.

27. Golomb BA, Evans MA. Statin adverse effects: a review of the literature and evidence for a mitochondrial mechanism. Am J Cardiovasc Drugs. 2008;8(6):373-418.

28. ICD9data.com [homepage on the Internet]. Diagnosis code 272.0. Pure hypercholesterolemia; ICD; 2015. Available from: http://www.icd9data. com/2015/Volume1/240-279/270-279/272/272.0.htm. Accessed July 10, 2017.

29. World Health Organization [homepage on the Internet]. ICD-10 Version: 2015. WHO. Available from: http://apps.who.int/classifications/ icd10/browse/2015/en\#/E70-E90. Accessed July 10, 2017.

30. Coding-pro.com [homepage on the Internet]. Pure hypercholesterolemia. ICD-10-CM tabular list. Available from: http://www.coding-pro.com/ icd-10/code_details.php?icd_code=E780. Accessed July 10, 2017.

31. Guyton JR, Bays HE, Grundy SM, Jacobson TA. The National Lipid Association Statin Intolerance Panel. An assessment by the Statin Intolerance Panel: 2014 update. J Clin Lipidol. 2014;8(3 Supp1):S72-S81. 
32. Schulman KL, Lamerato LE, Dalal MR, et al. Development and validation of algorithms to identify statin intolerance in a US administrative database. Value Health. 2016;19(6):852-860.

33. Liu X, Kubilis P, Bussing R, Winterstein AG. Development of a refill pattern method to measure polypharmacy in administrative claims databases. Pharmacoepidemiol Drug Saf. 2016;25(12):1407-1413.

34. Bushnell GA, Sturmer T, White A, et al. Predicting persistence to antidepressant treatment in administrative claims data: considering the influence of refill delays and prior persistence on other medications. $J$ Affect Disord. 2016;196:138-147.

35. Taitel M, Fensterheim L, Kirkham H, Sekula R, Duncan I. Medication days' supply, adherence, wastage, and cost among chronic patients in Medicaid. Medicare Medicaid Res Rev. 2012;2(3): pii: mmrr.002.03.a04.

36. Amgen [homepage on the Internet]. Analyses of PCSK9 inhibitor prescription rejection rates demonstrate significant access barriers for appropriate patients. Amgen; 2017. Available from: https://www. amgen.com/media/news-releases/2017/03/analyses-of-pcsk9-inhibitorprescription-rejection-rates-demonstrate-significant-access-barriers-forappropriate-patients/. Accessed July 10, 2017.

37. Medscape [homepage on the Internet]. Wendling P. PCSK9 inhibitor access snarled in red tape, rejections. Medscape; 2017. Available from: http://www.medscape.com/viewarticle/877515. Accessed May 1, 2017.

38. Saeed A, Virani SS, Jones PH, Nambi V, Zoch D, Ballantyne CM. A simplified pathway to proprotein convertase subtilisin/kexin type 9 inhibitor prior authorization approval: A lipid clinic experience. J Clin Lipidol. 2017;11(3):596-599.

39. Navar AM, Taylor B, Fievitz E, et al. Early challenges for PCSK9 inhibitor prescriptions and patients: rejections and rates unfilled. Presentation to the American College of Cardiology 66th Annual Scientific Session and Expo; March 19; 2017; Washington, DC.

40. BlueCross BlueShield Federal Employee Program. Praluent prior approval request. BlueCross BlueShield; 2016 October 21. Available from: http://www.caremark.com/portal/asset/FEP_Form_Praluent.pdf. Accessed July 10, 2017.
41. Defense Health Agency Pharmacy Operations Division. MTF formulary management for PCSK9 inhibitor agent subclass. Defense Health Agency Pharmacy Operations Division; 2016. Available from: https:// health.mil/Reference-Center/Fact-Sheets/2017/02/21/FormularyManagement-for-PCSK9-Inhibitors. Accessed July 10, 2017.

42. CVS/Caremark. Specialty guideline management. Praluent (alirocumab). Caremark; 2016. Available from: http://www.caremark. com/portal/asset/Praluent_Policy.pdf. Accessed July 10, 2017.

43. United Healthcare Pharmacy, Clinical Pharmacy Programs. Prior authorization/medical necessity. Repatha (evolocumab). United Healthcare Pharmacy; 2016. Available from: https://www.unitedhealthcareonline.com/ccmcontent/ProviderII/UHC/en-US/Assets/ ProviderStaticFiles/ProviderStaticFilesPdf/Tools $\% 20$ and $\% 20$ Resources/Pharmacy\%20Resources/Clinical\%20Programs/PA_Med_ Nec_Repatha.pdf. Accessed July 10, 2017.

44. The Long-Term Intervention with Pravastatin in Ischaemic Disease (LIPID) Study Group. Prevention of cardiovascular events and death with pravastatin patients with coronary heart disease and a broad range of initial cholesterol levels. N Engl J Med. 1998;339(19): 1349-1357.

45. Cholesterol Treatment Trialists' (CTT) Collaboration, Fulcher J, O'Connell R, et al. Efficacy and safety of LDL-lowering therapy among men and women: meta-analysis of individual data from 174,000 participants in 27 randomised trials. Lancet. 2015;385(9976): 1397-1405.

46. Choudhry NK, Shrank WH. Four dollar generics - increased accessibility, impaired quality assurance. $N$ Engl J Med. 2010;363(20): 1885-1887.

47. Fairman KA, Curtiss FR. How do seniors respond to $100 \%$ cost-sharing for prescription drugs? Quality of the evidence underlying opinions about the Medicare Part D coverage gap. J Manag Care Pharm. 2011; 17(5):382-392.

48. Medical Marketing and Media Online [homepage on the Internet]. McCaffreyK. Amgenlaunchesnew RepathaDTCad.MM\&M;2017.Available from: http://www.mmm-online.com/campaigns/amgen-launchesnew-repatha-dtc-ad/article/642323/. Accessed July 10, 2017.
Therapeutics and Clinical Risk Management

\section{Publish your work in this journal}

Therapeutics and Clinical Risk Management is an international, peerreviewed journal of clinical therapeutics and risk management, focusing on concise rapid reporting of clinical studies in all therapeutic areas, outcomes, safety, and programs for the effective, safe, and sustained use of medicines. This journal is indexed on PubMed Central, CAS,

\section{Dovepress}

EMBase, Scopus and the Elsevier Bibliographic databases. The manuscript management system is completely online and includes a very quick and fair peer-review system, which is all easy to use. Visit http://www.dovepress.com/testimonials.php to read real quotes from published authors. 